\title{
Mobility of Polymers at the Air/Polymer Interface
}

\section{Tobias Kerle, Zhiqun Lin, Ho-Cheol Kim, and Thomas P. Russell*}

Silvio O. Conte National Center for Polymer Research, University of Massachusetts, Amherst, Massachusetts 01003

Received November 28, 2000; Revised Manuscript Received February 20, 2001

\begin{abstract}
In general, the relaxation of polymers from a nonequilibrium state will occur only if the polymer is above the bulk glass temperature. To examine the influence of a free surface on the mobility of polymer chains, the free surface of thick, artificially roughened polystyrene (PS) films ( $>10 \mu \mathrm{m}$ ) was studied. Each surface asperity of the PS film represents a nonequilibrium system with a large interfacial surface area. The Laplace pressure tends to suppress surface roughness, which can only occur if the polymer chains within the asperities are mobile. A partial relaxation of the surface asperities at temperatures well below the bulk glass temperature was observed. With increasing temperature the lateral size of the remaining surface asperities was found to increase, and the aspect ratio of the asperities decreased. However, a complete relaxation of the surface structures occurred only for temperatures comparable to or greater than the bulk glass transition temperature.
\end{abstract}

\section{Introduction}

While the static properties of polymeric materials at surfaces and interfaces have been the focus of much research, ${ }^{1-3}$ there have been far fewer studies of the dynamic properties. Adhesion, interdiffusion, and wear ${ }^{4-7}$ are strongly dependent on the dynamics or, more specifically, the mobility of the polymer chains at surfaces and interfaces. A key parameter that governs the mobility in polymers is the glass transition temperature $T_{g}$, which marks the crossover from an immobile, solidlike material to a liquid material showing flow behavior. Experiments by Keddie et al .8,9 indicated that the effective $T_{g}$ in thin film geometries, where sample properties are often governed by confinement effects and interactions, can strongly depend on the film thickness and the interfacial interactions. Keddie and co-workers, using spectroscopic el lipsometry, investigated the thickness dependence of the glass transition temperature in thin films of poly(methyl methacrylate) (PMMA) and polystyrene (PS) on solid substrates. They suggested that there was a surface layer of finite thickness with an increased mobility and thus a reduced glass transition temperature. As the temperature was increased to the bulk $T_{g}$, the thickness of this surface layer increased. According to Keddie et al., one expects the surface layer thickness to diverge as $T$ approaches $T_{g}$ from below. The effective glass transition temperature of the thin film will then depend on the ratio of film to surface layer thickness and on the surface and bulk glass transition temperatures. Forrest and co-workers, ${ }^{10-12}$ using ellipsometry and Brillouin light scattering, measured $T_{g}$ in freely standing thin PS films, thus imposing symmetric boundary conditions. Similar to the results reported by Keddie et al., Forrest and co-workers found a reduced effective glass transition temperature in polymeric thin film systems. Their measurements indicated a rather remarkable depression of the effective glass transition temperature for sample thicknesses approaching typical polymeric length scal es, i.e., the radius of gyration $R_{g}$.

Measurements of the free vol ume near the surface by de Maggio et al. ${ }^{13}$ and J ean et al., ${ }^{14}$ using positron annihilation, indicated that the glass transition temperature was lower than that in the bulk. Tanaka et al., ${ }^{15,16}$ Tsui et al., ${ }^{17}$ and Ge et al. ${ }^{18}$ used atomic force microscopy to study the viscoelastic properties of the near surface region $\left(\approx 2 R_{G}\right.$ from the surface). Tanaka et al. found that low molecular weight PS $\left(M_{w}=1700\right)$ showed a drop of the dynamic surface storage modulus by 1 order of magnitude in comparison to the bulk; however, no such effect was observable for $M_{w}>30000$. This is consistent with the observations of Liu et al.,19 who reported no suppression of $\mathrm{T}_{\mathrm{g}}$ at the free surface in comparison to the bulk, in a study of the relaxation of rubbed PS ( $\left.\mathrm{M}_{\mathrm{w}}=96000\right)$. Complete relaxation of the polymer was only observed at temperatures above $\mathrm{T}_{\text {g,bulk. }}$ From the shear and torsion moduli, Tsuil ${ }^{17}$ reported no differences in the molecular relaxation of the polymer near $\mathrm{T}_{\mathrm{g}}$ in the bulk and at the free surface. Thus, a comparison of the various experimental studies and results paints a rather confusing and inconclusive picture. Depending on the type of study and the technique applied to characterize the mobility, the mobility/glass transition temperature is either largely changed or indistinguishable from the bulk value. An excellent overview of the current development in this field of research can be found in the recent article by J ones. ${ }^{20}$

Similarly opposing observations on the mobility of chains at the polymer/air interface can also be found in the theoretical literature-even within the very same study. Doruker and Mattice, ${ }^{21}$ for example, in a simulation study on the mobility of the surface and interior of thin films composed of amorphous polyethylene, pointed out that the surface mobility depends critically on the precise definition of "mobility". Whereas the surface is more mobile than the bulk, in terms of decorrelation of the end-to-end vectors, or the apparent diffusivity, one finds even a mobility lower than the bulk value in terms of apparent diffusivity at longer times in a direction normal to the surface or in the normal component of the diffusion coefficient.

The physical origin of this potentially increased mobility of the surface layer is so far unclear and has been the subject of much discussion. ${ }^{12,22-27}$ The first theoretical arguments regarding the origin of the reduced $T_{g}$ were made by Mayes. ${ }^{23}$ Chain ends are enriched at interfaces in comparison to the bulk for 
entropic reasons. It was argued that this enrichment should reduce the polymer density in the interfacial region, which is supported by the simulation studies of Müller et al. ${ }^{25}$ and Rouault et al. ${ }^{26}$ The reduced density should consequently lead to an increased mobility of the chain segments and thus a reduction of $\mathrm{T}_{\mathrm{g}}$ in the surface region. A more quantitative description of this model predicts the following functionality for $\mathrm{T}_{\mathrm{g}}$

$$
\mathrm{T}_{\text {g,surface }}=\mathrm{T}_{\text {g,bulk }}-\mathrm{CN}^{-1 / 2}(\mathrm{a} / \mathrm{d})
$$

where $C$ is a material-dependent constant $(C=1100$ for $\left.\mathrm{PS}^{28}\right), \mathrm{N}$ is the number of monomers, $\mathrm{a}$ is the statistical segment length, and d is the thickness of the surface layer. A typical example illustrates the effect of this model. For PS with $\mathrm{M}_{\mathrm{w}}=100000$ and $\mathrm{a}$ segregated layer thickness of $\mathrm{d} \approx \mathrm{a}$, the model predicts a depression of $\mathrm{T}_{\mathrm{g}}$ in the surface layer by $\sim 36^{\circ} \mathrm{C}$. However, it has been argued ${ }^{19,23}$ whether an enrichment in chain ends is a realistic description of the experimental observations. As the depression of the surface $\mathrm{T}_{\mathrm{g}}$ scales with $\mathrm{N}^{-1 / 2}$, the model predicts a rapidly decreasing depression with increasing molecular weight, contrary to the experimental results of Keddie et al.8,9 For PS systems comparable to the ones studied by Keddie, ${ }^{8,9}$ F orrest, 10,11 and others, $15-17,19$ the predicted depression would be only about $1{ }^{\circ} \mathrm{C}$, which is smaller than the experimentally estimated values. Consequentially, the fraction of chain ends at the surface is too small to have a drastic impact on the density and, thus, the mobility at the polymer/air surface.

Another possible origin for the increased mobility was recently suggested by de Gennes. ${ }^{22}$ It was argued that there are two competing motions in polymeric systems: standard motions, controlled by the free volume, which are independent of the chain length, and collective motions which require, except for the end groups, a weaker free volume. Because of the end group hindrance, it is expected that the standard motion dominates in the bulk. However, in films thinner than the coil size the collective motion might become the dominant process. For the collective motion of a loop, chain end effects should become negligible. Thus, the rel evant scale is not given by the overall polymerization index $\mathrm{N}$ but by the length of a typical loop starting at the surface and reaching deep into the film. Qualitatively, this idea of a competition between two melting processes can explain the observed reduction of $\mathrm{T}_{\mathrm{g}}$ in the near surface region. However, the quantitative numerical predictions of this model are poor.

Here we introduce a novel technique to study the mobility of polymers at the polymer/air interface. Artificially rough model surfaces were used to investigate the surface tension driven decay of polymer surface asperities. The flattening of a liquid surface driven by the Laplace pressure is a well-studied phenomenon for the case of fully liquid systems. ${ }^{29,30}$ Orchard et al. showed, nearly 40 years ago, that in the case of thick liquid films the characteristic time for flattening is given as $\eta \lambda / \gamma$, where $\eta$ is the viscosity, $\lambda$ is the wavelength, and $\gamma$ is the interfacial tension of the liquid.27 The experiment reported here utilizes this effect to probe the mobility of polymeric systems at $\mathrm{T}<\mathrm{T}_{\mathrm{g}}$ on typical polymeric length scales, i.e., tens of nanometers. A somewhat similar idea was recently employed by Hamdorf and J ohannsmann, ${ }^{31}$ who studied the surface tension driven decay of imprinted micron-sized corruga-
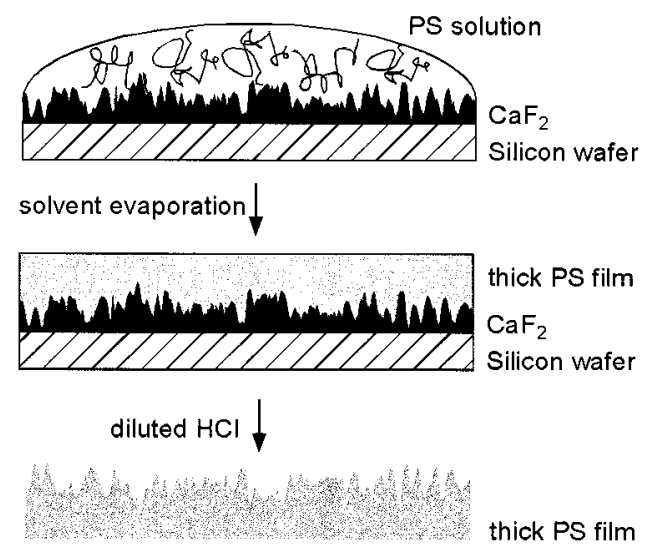

Figure 1. Sketch of the sample preparation technique used. Thick polystyrene films (d >10 $\mu \mathrm{m}$ ) were prepared on top of artificially rough $\mathrm{CaF}_{2}$ substrates by casting a toluene solution on the substrates (a) and subsequent evaporation of the solvent and annealing of the sample for 2 days at $\mathrm{T}_{\mathrm{A}}=170^{\circ} \mathrm{C}(\mathrm{b})$. A separation of the polymer film and the Si substrate was achieved by a selective dissolution of the $\mathrm{CaF}_{2} / \mathrm{Al}$ layer with dilute $\mathrm{HCl}$. Subsequently, the PS sample was transferred onto dei onized water to wash away the residual $\mathrm{CaF}_{2}, \mathrm{Al}$, and $\mathrm{HCl}$ and dried for 1 day at room temperature under vacuum $\left(10^{-1}\right.$ mbar) (c).

tion gratings. On this length scale it was observed that the near-surface moduli agree with the bulk values for highly entangled samples. However, the apparent stiffness increases by a factor of 10 compared to the bulk for chains shorter than the entanglement length. In our studies, the analysis of the temporal evolution of the ensemble of asperities of various sizes and aspect ratios allows a unique assessment of the mobility of chains under varying degrees of confinement over a large range of length scales. Our investigations indicate that there indeed exists a restricted mobility of chains at temperatures far below $\mathrm{T}_{\text {g,bulk. }}$ It was observed that even 40 ${ }^{\circ} \mathrm{C}$ below $\mathrm{T}_{\text {g.bulk }}$ surface asperities relaxed. However, these relaxations only occur when the system is sufficiently far from equilibrium. For all experimental temperatures lower than $\mathrm{T}_{\mathrm{g} \text {,bulk }}$ only a partial relaxation of the surface roughness was observable. Single asperities relaxed even for very long annealing times only down to an aspect ratio characteristic for the given annealing temperature. A series of experiments were also performed to evaluate whether entropy (chain confinement) or enthalpy (interfacial tension) is the origin of this increased, but restricted, mobility.

\section{Experimental Section}

Materials. Narrow molecular weight distribution samples of polystyrene (PS) of molecular weight $\mathrm{M}_{\mathrm{w}}=9.6 \times 10^{4}$ and $M_{w}=3 \times 10^{6}$ were used. The polymers were purchased from Polymer Labs (Church Stretton, UK) and characterized by the manufacturer with a polydispersity of $\mathrm{M}_{\mathrm{w}} / \mathrm{M}_{\mathrm{n}}=1.04$ and $\mathrm{M}_{\mathrm{w}} /$ $M_{n}=1.03$, respectively. The bulk $T_{g}$ of both polymers is in the range $105-110{ }^{\circ} \mathrm{C}$. Calcium fluoride $\left(\mathrm{CaF}_{2}\right)(99.9 \%$ pure) was obtained from Aldrich. The toluene (analytical grade) and $\mathrm{HCl}$ were both purchased from EM Science (Gibbstown, NJ ).

Sample Preparation. Polystyrene samples with a controlled surface roughness were generated by applying a simple replication technique, as schematically shown in Figure 1 . In a first step $\mathrm{CaF}_{2}$ was evaporated onto a chemically polished silicon wafer. This results in a surface with a well-controlled, laterally random roughness with a Gaussian distribution in height about a mean value, as described in detail elsewhere. ${ }^{32}$ The topography is characterized by an rms surface roughness of a few nanometers. Single surface asperities (peaks) extend laterally, depending on the evaporation conditions, typically 

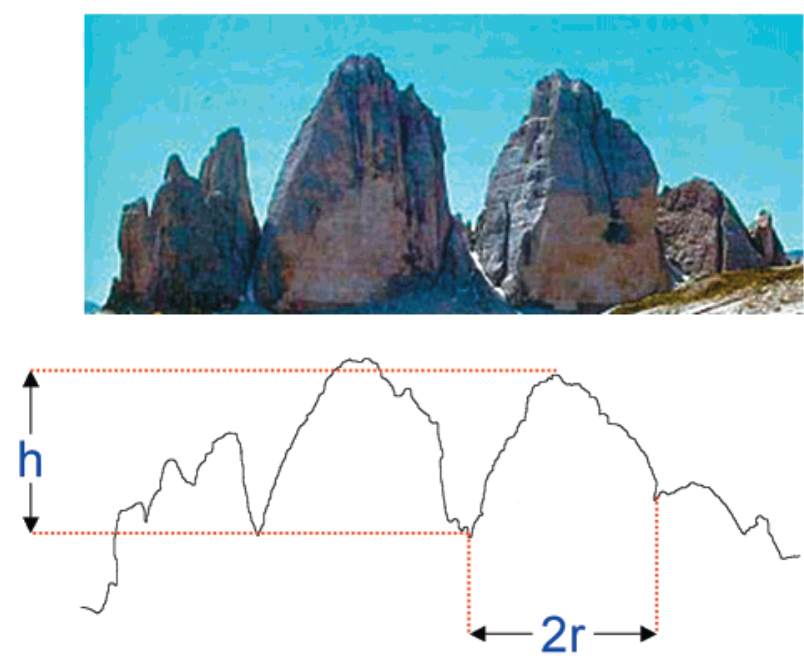

Figure 2. Line scan of a 3-dimensional topography indicating the definition of the key variables measured by the custombuilt analysis software: peak height (h) and peak base radius (r).

over a few tens of nanometers. Subsequently, a thin layer of aluminum is evaporated on top of the $\mathrm{CaF}_{2}$ layer. This results in a surface onto which polymer films can be easily prepared, while preserving the nature of the roughness.

Thick polystyrene films ( $d>10 \mu \mathrm{m}$ ) were prepared on top of these artificially rough substrates by casting a toluene solution (10 mg of PS per $1 \mathrm{~mL}$ of solvent) onto the substrates (Figure 1a), evaporating the solvent and annealing the sample for 2 days at $\mathrm{T}_{\mathrm{A}}=170{ }^{\circ} \mathrm{C}$, well above $\mathrm{T}_{\text {g,bulk }}$ (Figure 1b). A separation of the polymer film and the Si substrate was achieved by a selective dissolution of the $\mathrm{CaF}_{2} / \mathrm{Al}$ layer with dilute $\mathrm{HCl}$. Subsequently, the PS sample was transferred onto deionized water to wash away the residual $\mathrm{CaF}_{2}, \mathrm{Al}$, and $\mathrm{HCl}$ and dried for 1 day at room temperature under vacuum $\left(10^{-1}\right.$ mbar). Control measurements using X-ray photoelectron spectroscopy measurements indicated no residual traces of $\mathrm{Ca}, \mathrm{F}$, $\mathrm{Al}$, or $\mathrm{Cl}$ on the rough PS surfaces after washing and drying. The dried polystyrene films were mounted on top of fresh silicon wafers with the rough side facing air (Figure 1c) and stored in a desiccators until used.

Experimental Techniques. Surface topography measurements were performed on a Digital Instrument Dimension 3000 atomic force microscope in tapping mode with etched silicon tips (Nanoprobe). The spring constant of the tips was $\mathrm{k}=40-66 \mathrm{~N} / \mathrm{m}$ (manufacturer specification). The analysis of the temporal evolution of the surface structure was carried out with the standard instrument software and custom-made software allowing the full statistical assessment of the surface asperities. The software analyzed the AFM topography data in two steps. First, the program generated a list of the peak positions by finding all $\mathrm{x}-\mathrm{y}$ coordinates that are higher than all their direct neighbors. In a second step each peak was measured as indicated in the sketch shown in Figure 2. By analyzing the surrounding of a given peak center, the program evaluated the size of the peak, as characterized by the peak height (the vertical distance between the lowest point of the peak and the crest) and the peak base radius [(projected area/ $\pi)^{1 / 2}$. The output file consisted of a list of the peak coordinates, $h, r$, and the aspect ratio $h / r$, thus allowing a detailed statistical analysis of these parameters.

\section{Results}

Thermal Evolution of Artificially Rough PS Surfaces. AFM measurements of a PS sample with controlled surface roughness ( 2.5 by $2.5 \mu \mathrm{m}^{2}$ ) showed an initial, intrinsic rms surface roughness of about 4.5 $\mathrm{nm}$. Subsequently, the sample was anneal ed for $2 \mathrm{~h}$ at $\mathrm{T}_{\mathrm{A}}=40{ }^{\circ} \mathrm{C}$ under vacuum and remeasured. This anneal-measurement cycle was repeated for increasing $\mathrm{T}_{\mathrm{A}}$ up to well above $\mathrm{T}_{\mathrm{g} \text {,bulk. }}$. Fi gure 3 shows characteristic AFM images of the thermal evolution of this sample on equal scales (x, y: $2.5 \mu \mathrm{m}$; $\mathrm{z}: 30 \mathrm{~nm}$ ). An inspection of these micrographs shows a structural reorganization of the surface topography at temperatures well below $\mathrm{T}_{\text {g,bulk. With increasing temperatures, coarser structures }}$ of decreasing amplitude are observed. A detailed analysis of this set of experiments is shown in Figure 4, where the rms roughness of representative areas of 2.5 by 2.5 $\mu \mathrm{m}^{2}$ is plotted as a function of the annealing temperature. The observed functionality is reasonably welldescribed by a hyperbolic tangent function, centered at a temperature $\mathrm{T}_{0}$. In the temperature range between room temperature and $70{ }^{\circ} \mathrm{C}$ one observes only small changes in the rms surface roughness. With increased temperature $\left(75-105^{\circ} \mathrm{C}\right)$ one observes a gradual relaxation of the surface roughness to about a value of 0.5 $\mathrm{nm}$, which is comparable to that obtained for samples annealed well above $T_{g, b u l k}$. The inset of Figure 4 shows a comparison of these data with an earlier study on mobility of PS segments by Liu et al., ${ }^{19}$ where the dichroic ratio of rubbed PS surfaces was studied as a function of temperature by means of near-edge X-ray absorption fine structure (NEXAFS). Both sets of data indicate an onset of relaxation from a nonequilibrium state at a temperature of about $80^{\circ} \mathrm{C}$. However, it should be noted that the exact onset for relaxations in the case of the rough PS surfaces depends somewhat on the initial preparation conditions. Control experiments showed that with increasing size of the initial asperities, e.g., due to a higher $\mathrm{CaF}_{2}$ evaporation rate, $\mathrm{T}_{0}$ shifts to slightly higher values (up to $5^{\circ} \mathrm{C}$ in the most extreme case, where the initial surface roughness was about twice as high as in the case shown here).

Temporal Evolution of Artificially Rough PS Surfaces at a Constant Temperature. Time-dependent studies of the change of the surface topography of the rough PS samples were carried out over the temperature range from $65{ }^{\circ} \mathrm{C}$ to $\mathrm{T}_{\text {g,bulk. }}$. As repeated annealing and quenching of a single sample might lead to a rather undefined time lag associated with the sample warming to the anneal ing temperature, a set of samples with comparable intrinsic surface topographies was prepared by dividing a large $\left(5\right.$ by $5 \mathrm{~cm}^{2}$ ) film into smaller pieces. AFM scans of those pieces (not shown) indicated comparable rms roughnesses and showed similarly sized surface asperities for all samples. Subsequently, these samples were annealed at a fixed temperature $\mathrm{T}_{\mathrm{A}}$. In the following a typical set of results obtained for $\mathrm{T}_{\mathrm{A}}=75{ }^{\circ} \mathrm{C}$ will be discussed. A first impression of the temporal evolution at this temperature can be obtained from the linescans shown in Figure 5 . The line scans were generated by choosing a random scan line of a 2.5 by $2.5 \mu \mathrm{m}^{2}$ AFM surface map of a nonannealed sample (a) and samples annealed for $t_{A}=$ $10,60,300$, and 2880 min (b-e), respectively, on equal scales. A comparison of the nonannealed sample (a) and the sample annealed for $10 \mathrm{~min}$ at $75^{\circ} \mathrm{C}$ (b) shows a rapid disappearance of smaller sized asperities but no significant change in the amplitude of the asperities. With further annealing (10-300 $\mathrm{min}$ ) a decrease of the average asperity height is observed. However, no significant changes are observed for longer annealing times; e.g., the image obtained after $t_{A}=2880$ min (e) shows similarly sized asperities as the one obtained after $t_{A}=300$ min (d). A clearer picture of the relaxation 
not annealed

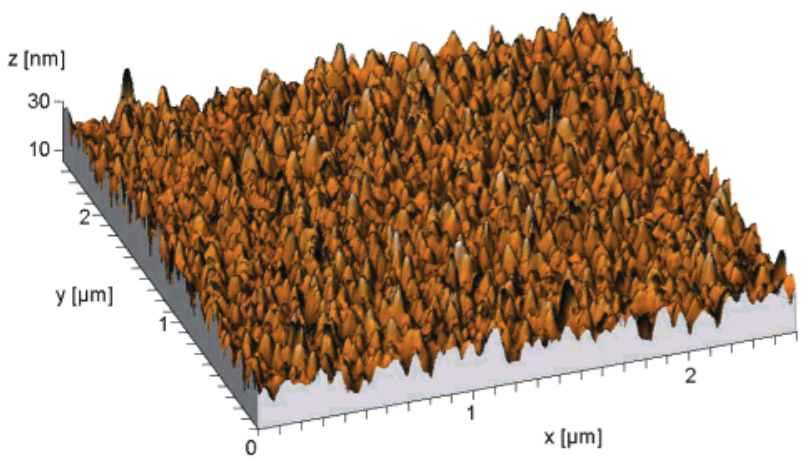

$\mathrm{T}=90^{\circ} \mathrm{C}$

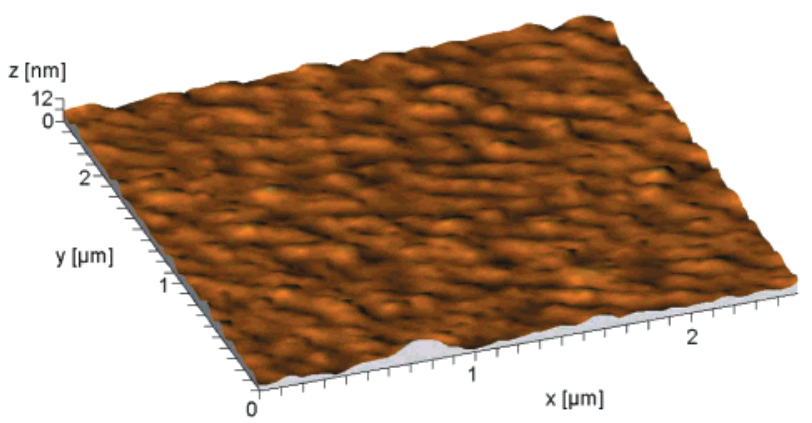

\section{$\mathrm{T}=80^{\circ} \mathrm{C}$}

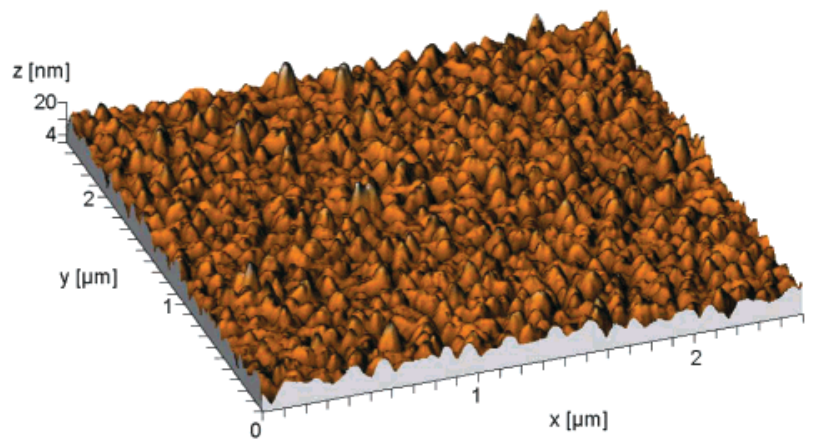

$\mathrm{T}=100^{\circ} \mathrm{C}$

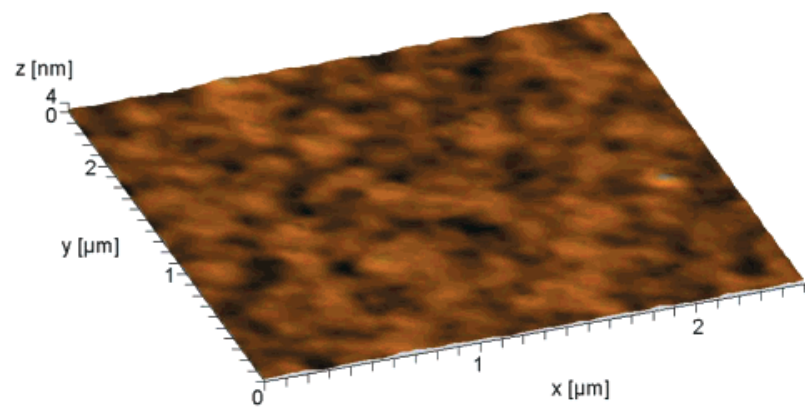

Figure 3. Typical AFM topography scans of the identical artificially rough PS sample before annealing and after annealing for $2 \mathrm{~h}$ each at increasing temperatures: 80,90 , and $100^{\circ} \mathrm{C}$. With increasing temperature coarser structures of decreasing amplitudes are observed.

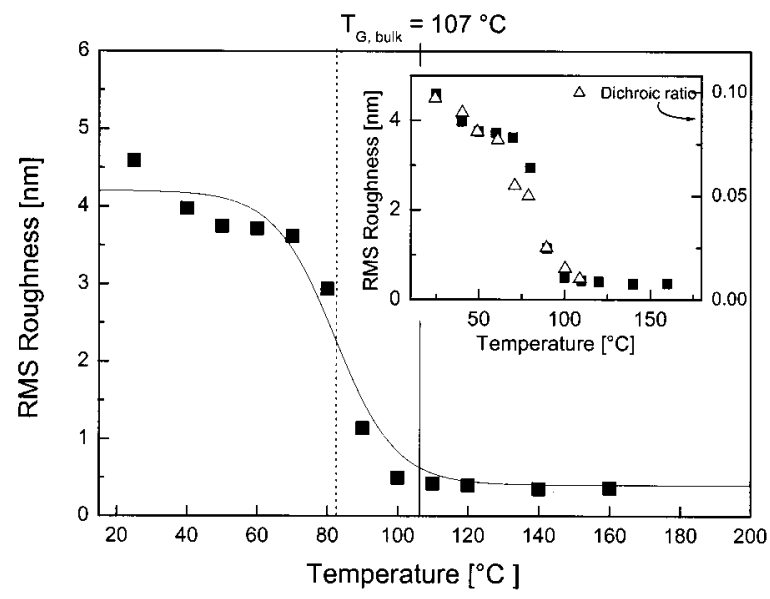

Figure 4. Thermal evolution of the rms surface roughness of representative 2.5 by $2.5 \mu \mathrm{m}^{2}$ areas of the sample shown in Figure 3, as obtained by AFM. With increased temperature one observes a gradual relaxation of the surface roughness to about a value of $0.5 \mathrm{~nm}$, which is comparable to that obtained for samples annealed well above $T_{\text {g,bulk. The inset shows a }}$ comparison of these data with an earlier study on mobility of PS segments by Liu et al., ${ }^{18}$ where the dichroic ratio of rubbed PS surfaces was studied as a function of temperature by means of near-edge X-ray absorption fine structure (NEXAFS).

of the surface roughness may be obtained from a statistical analysis of the surface asperities, as described in detail in the Experimental Section. Figure 6 shows as an example the temporal evolution of the peak height distribution function of three of the samples shown in Figure 5 with $t_{A}=0,10$, and $2880 \mathrm{~min}$. All three

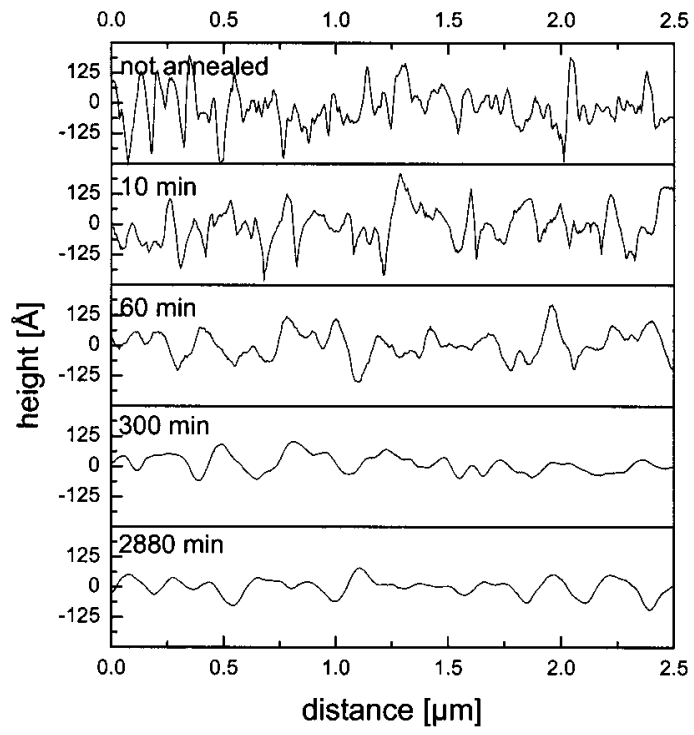

Figure 5. Typical AFM line scans of artificially rough PS samples of comparable initial roughness, annealed for various times (not annealed, 10,60, 300, and $2880 \mathrm{~min}$ ) at $\mathrm{T}=75^{\circ} \mathrm{C}$.

distributions are well described by Gaussian distribution functions. A comparison of the nonannealed sample (squares) and the sample annealed for $10 \mathrm{~min}$ (open circles) shows, similar to the observation based on the line scans, no significant change in the typical asperity height. However, the number of peaks, i.e., the integral of the distribution function, drops by roughly a factor of 2. An analysis of the peak radius distribution function (inset) shows that this apparent loss of peaks is ac- 


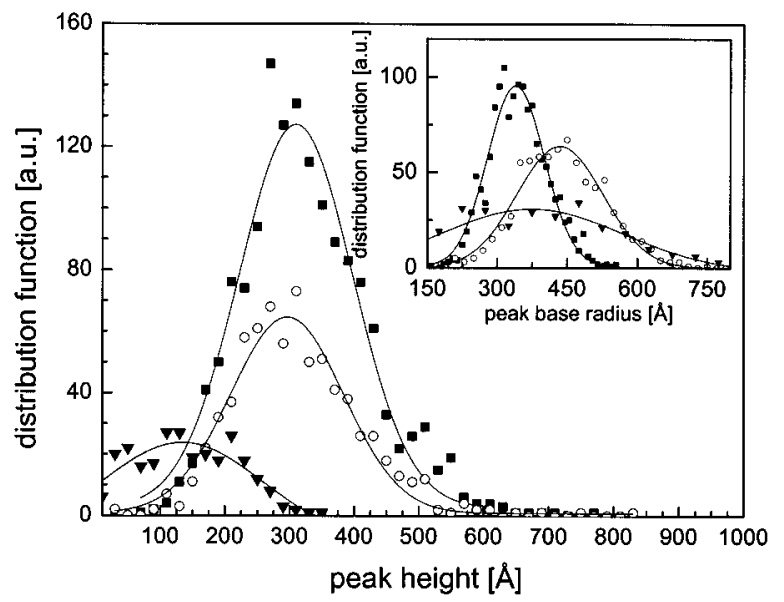

Figure 6. Temporal evolution of the peak height distribution function of three of the samples shown in Figure 5 with $t_{\mathrm{A}}=$ 0 (squares), $10 \mathrm{~min}$ (circles), and $2880 \mathrm{~min}$ (triangles). All three distributions are well described by Gaussian distribution functions.

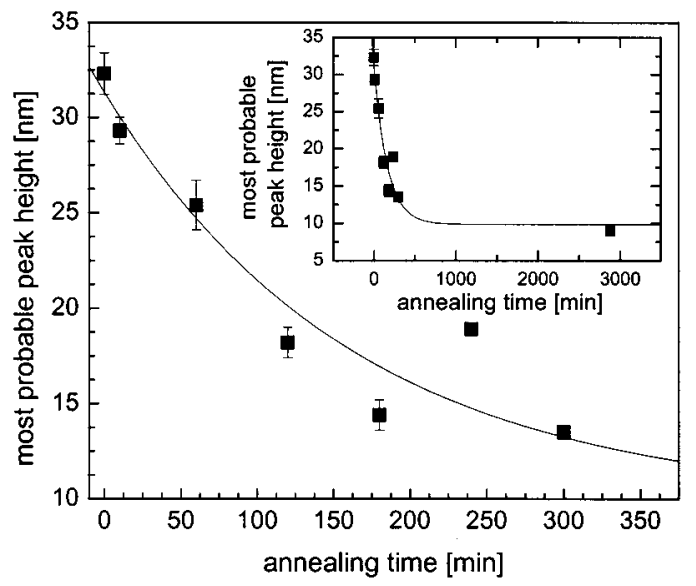

Figure 7. Temporal evolution of the most probable peak height, as defined by the center of the fitted Gaussian distribution functions (Figure 6), for the $75^{\circ} \mathrm{C}$ series (Figure 5). The error bar indicates the statistical deviation obtained from analyzing different samples prepared identically.

companied by an increase of the lateral size of the remaining peaks. This is, most likely, caused by the annealing of smaller substructures. Further annealing results in a clear decrease of the typical peak height and a further reduction in the number of surface asperities. It is worthwhile noting that the typical lateral dimension of the single peaks is, within the scatter, constant. Figure 7 corroborates the results obtained from the analysis of the peak height distribution functions of the $75{ }^{\circ} \mathrm{C}$ series. The data show the most probable peak height, i.e., the center of the fitted Gaussian distribution, as a function of the annealing time. The error bar indicates the statistical deviation of this value as obtained from independent measurements on identically prepared surfaces. The solid line shows an empirical fit of an exponential decay $\left(y=y_{0}\right.$ $+\mathrm{Ae}^{-\mathrm{t} / \tau}$ ), which describes the experimental data reasonably well. The time constant obtained from the fit is $\tau$ $=163 \mathrm{~min}$. The inset of the figure shows the long time devel opment of the average peak height. After the initial drop, as shown in the main figure, the typical peak height decreases only slightly to a value of about $10 \mathrm{~nm}$ and does not relax any further. A similar behavior can be observed in the temporal evolution of the peak aspect ratio (Figure 8). The expectation value for the aspect

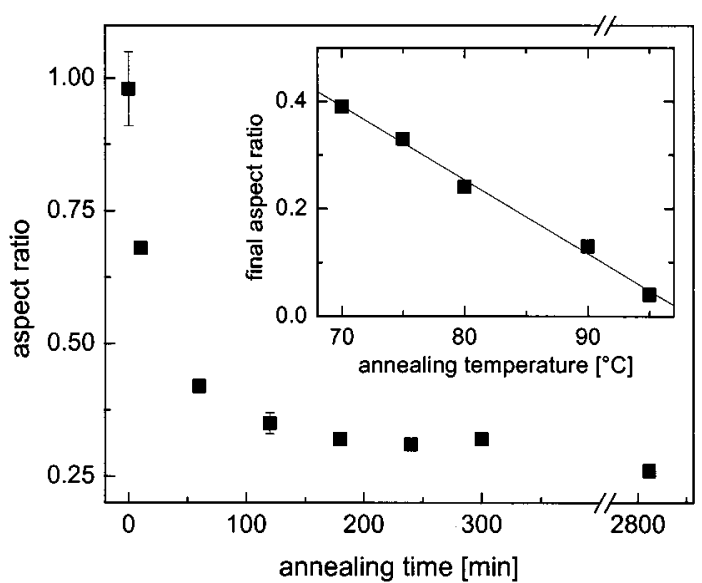

Figure 8. Temporal evolution of the most probable aspect ratio, as defined by the center of the fitted Gaussian distribution functions (Figure 6), for the $75^{\circ} \mathrm{C}$ series (Figure 5). The error bar indicates the statistical deviation obtained from analyzing different samples prepared identically. Experiments at different annealing temperatures $\mathrm{T}_{\mathrm{A}}$ indicate that the value at which the aspect ratio levels off is predominantly a function of $\mathrm{T}_{\mathrm{A}}$ and appears to be independent of the initial preparation conditions. The inset shows this plateau value as a function of $T_{A}$. The solid line in the figure indicates an empirical fit of a linear decay, which describes the data well.

ratio, as defined by the center of the fitted Gaussian function, drops from an initial value of about 1 to a constant level of 0.25 . The error bar indicates again the statistical deviation obtained from analyzing different samples prepared identically. Experiments at different annealing temperatures $\mathrm{T}_{\mathrm{A}}$ indicate that the value at which the aspect ratio levels off is predominantly a function of $T_{A}$ and appears to be independent of the initial preparation conditions. The inset of Figure 8 shows this plateau value as a function of $\mathrm{T}_{\mathrm{A}}$. The solid line in the figure indicates an empirical fit of a linear decay, which describes the data well. It is interesting to note that the fit intersects the abscissa at $\sim 98{ }^{\circ} \mathrm{C}$, essentially $T_{\text {g,bulk. }}$.

Comparative Study of Molecular Weight Effects. To evaluate the influence of molecular weight on the mobility at the surface of thick PS films, comparative studies with two different molecular weights were carried out. For this study samples with comparable intrinsic surface roughnesses and similarly sized surface asperities were prepared from PS with a molecular weight of $9.6 \times 10^{4}$ and $3 \times 10^{6}$, respectively. Although both materials have comparable bulk glass transition temperatures, it is well-known that the viscosity of PS above $T_{\text {g,bulk }}$ scales as $\eta \propto M_{w}{ }^{3.4}$ (for $M_{w}>M_{c}$ ), i.e., the higher molecular weight material being less mobile in the bulk. Figure 9 shows representative AFM scans for the two materials nonannealed and annealed at $\mathrm{T}_{\mathrm{A}}=$ $90{ }^{\circ} \mathrm{C}$ for 300 and $2880 \mathrm{~min}$, respectively. Comparison of the thermal evolution of the surface roughness of the two materials does not show any dramatic differences in the relaxation pattern. At equal times the samples show comparable sized surface asperities. A more detailed analysis of the temporal evolution of the surface topography for $\mathrm{T}_{\mathrm{A}}=90{ }^{\circ} \mathrm{C}$ can be seen in Figure 10, where the rms surface roughness for both systems is shown as a function of the annealing time. Within the scatter of the data no differences between the two are found. Both systems show an initial rapid drop of the rms surface roughness to a constant value of about 2 $\mathrm{nm}$, which does not relax any further, even for very long 


$$
M_{w}=96,000
$$

\section{not annealed}

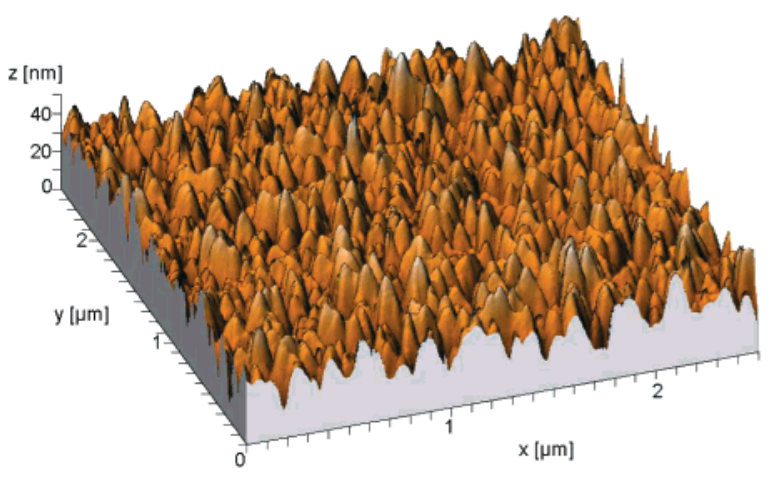

\section{$\mathbf{t}=\mathbf{3 0 0} \min$}

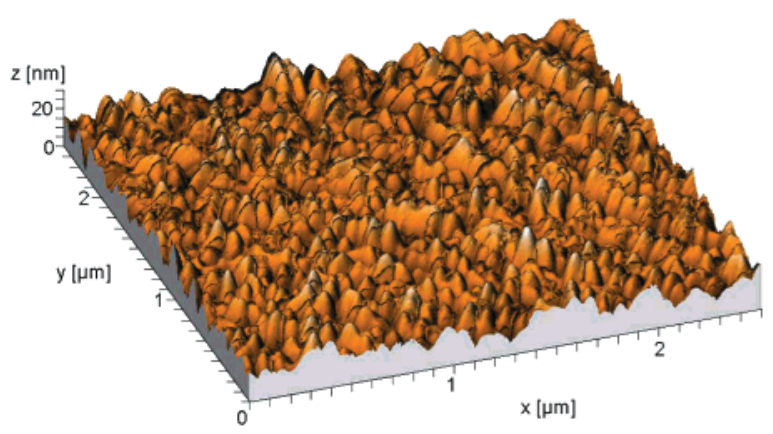

\section{$\mathbf{t}=\mathbf{2 8 8 0} \min$}

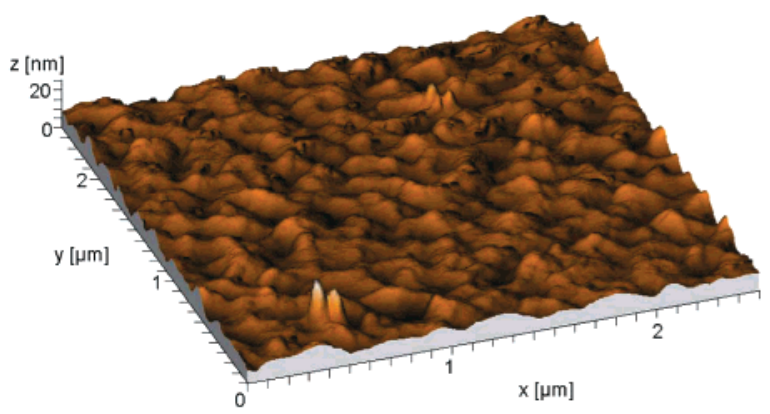

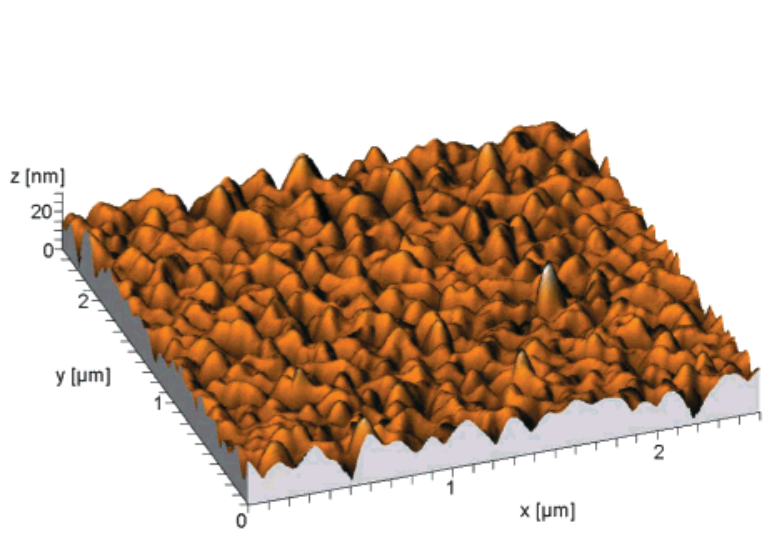

$M_{w}=3,000,000$
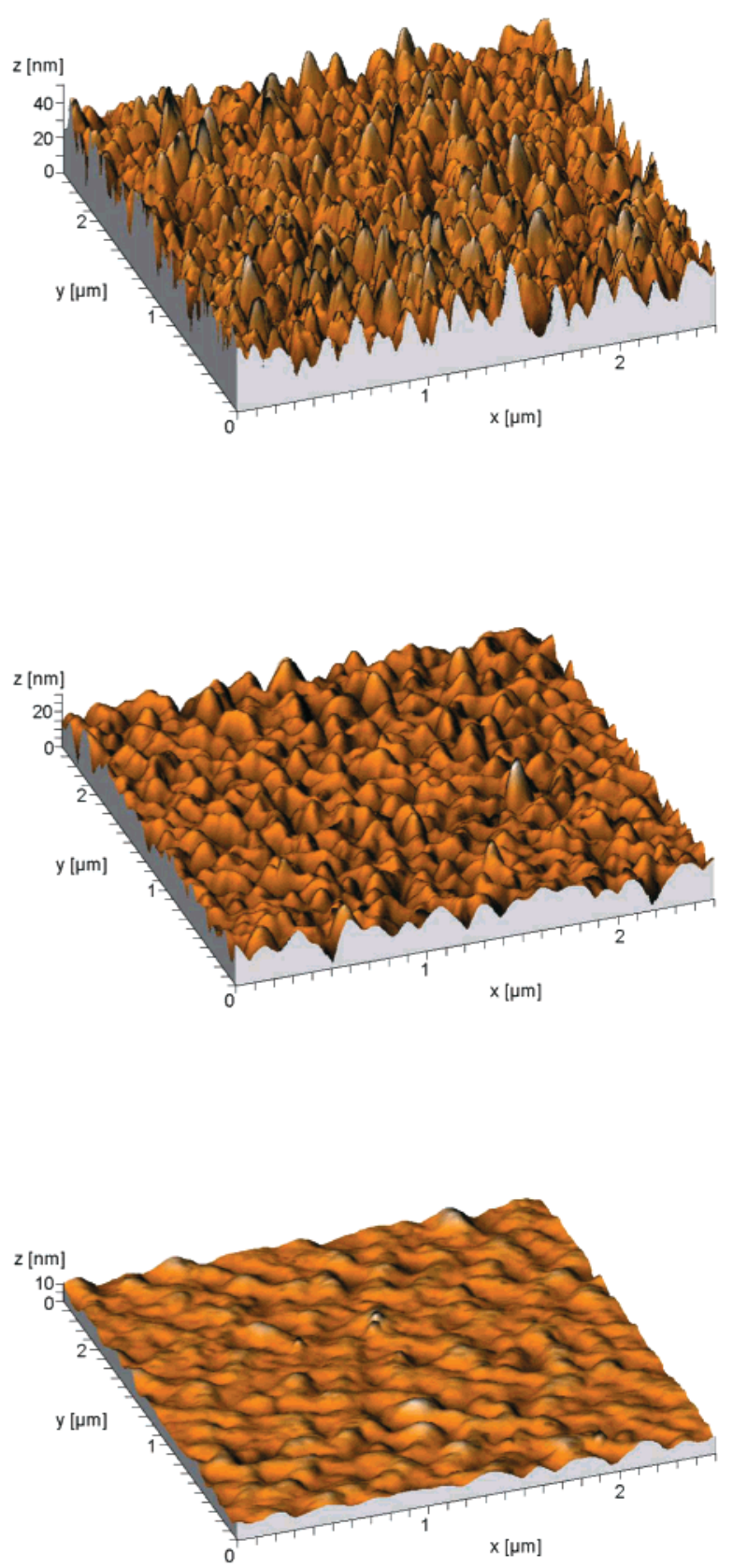

Figure 9. Representative AFM scans of artificially rough PS samples with comparable intrinsic surface roughnesses $\left(M_{w}=9.6\right.$ $\times 10^{4}$ and $3 \times 10^{6}$, respectively) nonannealed and annealed at $\mathrm{T}_{\mathrm{A}}=90^{\circ} \mathrm{C}$ for 300 and $2880 \mathrm{~min}$. A comparison of the thermal evolution of the surface roughness of the two materials does not show any dramatic differences in the relaxation pattern. At equal times the samples show comparable sized surface asperities.

annealing times. A qualitatively similar observation can be made from the data shown in the inset, where the typical aspect ratio for the two systems is plotted as a function of annealing time. Both systems drop over a comparable time scale to the same finite plateau value. The observed plateau value of 0.1 compares well to the data obtained in the earlier studies, shown in the inset of Figure 8.

Variation of Interfacial Interactions. In a last set of experiments the influence of interfacial interactions on the relaxation process was probed. For these experi- ments a set of PS $\left(\mathrm{M}_{\mathrm{w}}=9.6 \times 10^{4}\right)$ samples with comparable intrinsic roughnesses were prepared and divided into three batches. Macroscopic drops of either oligomeric PDMS or PI were placed on the samples of batch 2 and 3, to act as a capping layer. Subsequently, samples from all three batches were annealed for various times at $\mathrm{T}_{\mathrm{A}}=85^{\circ} \mathrm{C}$. After annealing, the PDMS and $\mathrm{PI}$ capping layers were removed with a selective solvent (heptane), and the samples were scanned by AFM. Control measurements of nonannealed samples where the PDMS and PI layers were removed indicated 


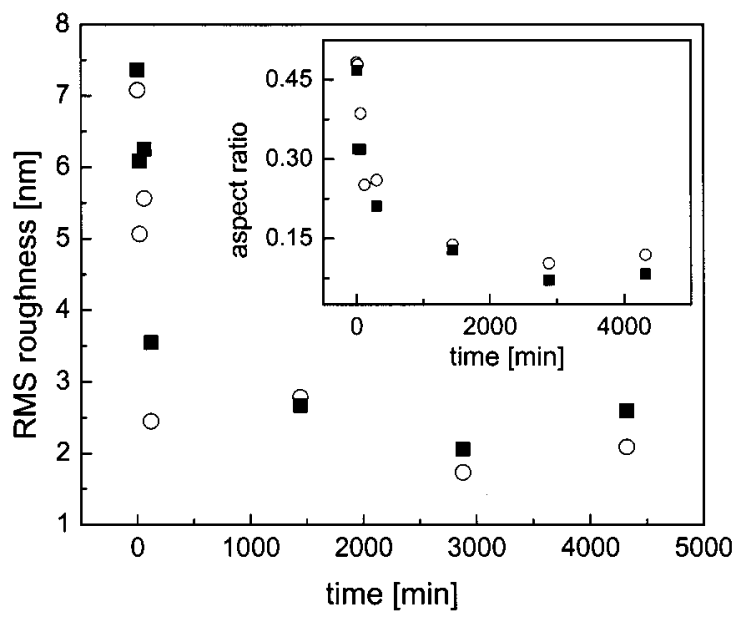

Figure 10. Temporal evolution of the rms surface roughness for both systems shown in Figure 9. The inset shows the typical aspect ratio for the two systems as a function of the annealing time.

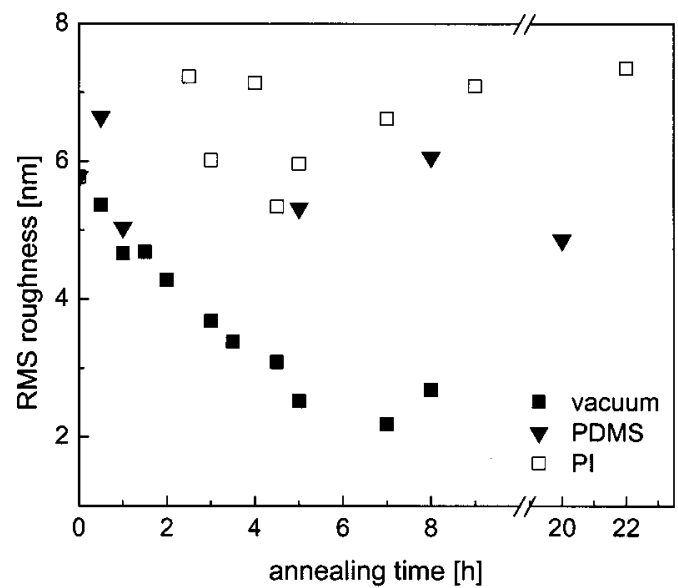

Figure 11. Temporal evolution of the rms surface roughness of artificially rough PS samples with comparable intrinsic surface roughnesses annealed under different boundary conditions (i.e., PS/vacuum, PS/PDMS, and PS/PI)

that the capping layer can be fully removed without modifying the underlying structure. Figure 11 shows the results of this study in terms of the rms surface roughness as a function of the annealing time. While the samples annealed under vacuum show a behavior comparable to that presented earlier, i.e., a rapid partial relaxation only, both the PI and the PDMS capped samples behave completely differently. Although the scatter in the data is in both cases slightly higher than in the polymer/vacuum case, no or only a very slight reduction of the surface roughness as a function of the annealing time is observable.

\section{Discussion}

The principal finding of this study is the observation of a partial relaxation of nanoscopic surface asperities of rough PS samples at temperatures well below $T_{\text {g,bulk. }}$. Both the rate and extent of the relaxation depend critically on the annealing temperature. These observations indicate an unexpected feature size and aspect ratio dependent mobility in polymeric systems well

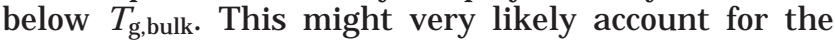
controversial results reported so far on the issue of mobility of polymers at free interfaces. A reasonable starting point for the discussion of our observations are the experimental studies and models proposed by Keddie et al ${ }^{8,9}$ and F orrest et al. ${ }^{10-12}$ Keddie and co-workers measured the thickness dependence of the glass transition temperature of thin films of PMMA and PS on solid substrates by using spectroscopic ell ipsometry to detect the discontinuity in thermal expansivity occurring at $T_{g}$. They observed that the effective $T_{g}$ of their thin film samples depends on the film thickness $\mathrm{d}$. It was suggested that this behavior is caused by a liquidlike layer of polymer at the air surface, whose size $\xi$ diverges as $\mathrm{T}_{\mathrm{g}}$ is approached from bel ow as

$$
\xi=\mathrm{A}\left(1-\frac{\mathrm{T}}{\mathrm{T}_{\mathrm{g}, \mathrm{bulk}}}\right)^{-v}
$$

where $\mathrm{A}$ and $v$ are material-dependent fit parameters and $T_{\text {g,bulk }}$ is the bulk glass transition temperature. This results in an effective glass transition temperature of the thin film system of

$$
\mathrm{T}_{\mathrm{g}}(\mathrm{d})=\mathrm{T}_{\mathrm{g}, \mathrm{bulk}}\left(1-\left(\frac{\mathrm{A}}{\mathrm{d}}\right)^{1 / \mathrm{v}}\right)
$$

A more refined approach was recently presented by Forrest and Mattson, ${ }^{12}$ who based their model on earlier experiments of Forrest et al. ${ }^{10,11}$ of $\mathrm{T}_{\mathrm{g}}$ for thin, freely standing polystyrene films. To describe the experimental results, Forrest and Mattson incorporated the idea of a characteristic length scale for cooperative dynamics into a simple layer model. Similar to Keddie and coworkers, they considered the film to have a region near the free surface with enhanced mobility due to the release of steric constraints. In analogy to eq 3, they related the effective glass transition temperature $T_{g}(d)$ of the thin films to the film thickness $d$ as

$$
T_{g}(d)=T_{g, \text { bulk }}+\frac{2 \xi\left(T_{g}(d)\right)\left(T_{g, \text { surf }}-T_{g, \text { bulk }}\right)}{d}
$$

where $T_{g, \text { surf }}$ is the glass transition temperature of the surface region of the size $\xi(T)$. Within the framework of their model two possible parametrizations of the temperature dependence of this length were exami ned: $\xi(T)=r_{0}+\alpha\left(T_{\text {ons }}-T\right)^{\gamma}$ and, al ternatively, $\xi(T)=\xi\left(T_{g}\right)$ $+\alpha\left(T_{g, b u l k}-T\right)^{\gamma}$ where $r_{0}$ is the average distance between monomer units, $T_{\text {ons }}$ is the onset temperature for cooperative motion, $\xi\left(T_{q}\right)$ is the size of the cooperativity length at the glass transition temperature, and $\alpha$ and $\gamma$ are fit parameters. Both parametrizations were found to describe the experimental data equally well. An analysis of a typical experiment (thin films of PS, $\mathrm{M}_{\mathrm{w}}=347000$ ) using the second form of parametrization yields $\mathrm{T}_{\mathrm{g} \text {,surf }}=305 \pm 7 \mathrm{~K}, \alpha=1.4 \pm 0.17, \gamma=0.95 \pm$ 0.15 , and $\xi\left(T_{q}\right)=26 \pm 21 \AA$. This implies a pseudolinear scaling of $\left(\xi(T)-\xi\left(T_{g}\right)\right)$ with $\left(T_{\text {g,bulk }}-T\right)$ and a value of the order of a few nanometers for $\xi(T)$ for this system over the temperature range $(275-375 \mathrm{~K})$ investigated.

Both models assume that the typical extent of the liquidlike layer is a function of the annealing temperature only. However, this assumption is not consistent with the qualitative behavior observed in our experiments as will be discussed. We recall at this point some of the experimental findings of our study. When artificially rough PS surfaces were annealed at temperatures $\mathrm{T}<\mathrm{T}_{\text {g,bulk, }}$ a rapid partial relaxation of the roughness was observed. The extent and rate of this relaxation are a function of the annealing temperature. As T approaches $T_{g, b u l k}$ the annealing process occurs more 
rapidly, resulting in smoother surfaces. As the driving force for this relaxation process, i.e., the Laplace pressure, is expected to be, within a first approximation, temperature-independent, differences in the initial relaxation rate have to originate from differences in the mobility at the given temperatures. This is, so far, consistent with the assumption of the models. However, one would expect that if a liquidl ike surface layer exists whose extent is finite and only temperature dependent, a nonzero driving force for the relaxation, as given by the Laplace pressure, would ul timately result in the full relaxation of the surface roughness. This does not imply the relaxation to a zero rms but rather to the extent defined by the amplitude of the spectrum of capillary waves of the surface fluctuations. Typically, this would result in an rms surface roughness of about half a nanometer for an area of 2.5 by $2.5 \mu \mathrm{m}^{2}$. This is not observed in our experiments. All experiments carried out bel ow $T_{g, b u l k}$ clearly showed only a partial rel axation of the surface roughness, where the terminal degree of relaxation was observed to be a function of the annealing temperature. The extrapolation of our experimental data shown in Figure 8 indicates that a full relaxation of the surface roughness can only be expected for $\mathrm{T} \approx$ $\mathrm{T}_{\mathrm{g}, \mathrm{bulk}}$ and higher. Since there is still a finite driving force for further relaxation, this implies that the formerly mobile surface layer was rendered immobile as a consequence of the relaxation process itself. Thus, the mobility in the near surface region not only is a function of the temperature but also depends on parameters associated with the degree of nonequilibrium of the system.

Generally, both entropy and enthal py may affect the mobility of a given system. In the system investigated here a comparison of the initial asperity sizes and the characteristic length scale of the polymer, i.e., the radius of gyration $\mathrm{R}_{\mathrm{g}}$, indicates comparable length scales. This effectively results in a confinement of the chains, which has both entropic and enthalpic implications, as discussed in the following section. In terms of entropic arguments a very likely mechanism is a mobility induced by the relaxation of prestretched chains. It is well-known that stretched polymer chains can relax and contract far below $T_{\text {g,bulk. }}$ A classical textbook example for this behavior is the annealing of prestretched polystyrene sheets, where one observes a relaxation, i.e., a shrinking of the samples, at temperatures as low as $60-70^{\circ} \mathrm{C}$. This effect might be relevant for the system investigated here, as the initial confinement within the surface asperities can only be achieved by some degree of chain stretching. This effect should become stronger with the degree of confinement of the chains. Thus, one would expect a change of both rate and extent of the relaxation with an increase or decrease of the characteristic dimension of the polymer, i.e., $\mathrm{R}_{\mathrm{g}}$. This idea was tested in the experiments shown in the Experimental Section where two identically prepared sets of samples of $M_{w}=96000\left(R_{g}=12 \mathrm{~nm}\right)$ and $M_{w}=3000000\left(R_{g}=\right.$ $94 \mathrm{~nm}$ ) were annealed at $\mathrm{T}_{\mathrm{A}}=90{ }^{\circ} \mathrm{C}$ and compared in terms of their relaxation behavior. The experiment showed no clear differences in the rates or extents of relaxation of the two materials-an entropy-induced mobility of the near-surface region is thus highly unlikely. It should be noted that in bulk polymeric systems one expects the zero shear rate viscosity to scale as $\eta \sim \mathrm{M}_{\mathrm{w}}{ }^{3.4}$ for $\mathrm{T}>\mathrm{T}_{\mathrm{g}}$ and $\mathrm{N}>\mathrm{N}_{\mathrm{c}}$, where $\mathrm{N}_{\mathrm{c}}$ is the critical entanglement length. This is commonly at- tributed to the significant increase of the number of entanglements with increasing molecular weight. Thus, one might expect an effective slowing down of dynamic processes with higher molecular weight. However, this effect is unlikely to be relevant in the case discussed here, as the number of entangl ements per unit volume is likely to be reduced in the surface near region due to a break in symmetry at the polymer/air interfaces.

In terms of an enthalpic argument, a confinement of chains within the surface asperities implies that the chains within a single asperity are in contact with molecules of the surrounding media, i.e., air, which is a poor solvent for the polystyrene. This increase of contacts with a nonsolvent might lead to a liquidlike behavior of the surface layer. In our case one expects this effect to become weaker with a reduction of the Laplace pressure, which depends on both the interfacial tension and the amount of exposed surface area. This assumption was tested in the set of experiments, where three identically prepared PS samples were annealed under different boundary conditions, i.e., modified interfacial energies, at $\mathrm{T}=85^{\circ} \mathrm{C}$ and compared in terms of their relaxation behavior. It was observed that both the extent and rate of relaxation drop remarkably with decreasing interfacial energy-the sample configuration with the lowest interfacial energy, i.e., PS/PI, showed within the scatter no temporal evol ution of the surface roughness at all. While a certain slowing down of the relaxation processes is expected within the framework of the theory by Orchard et al., ${ }^{29}$ the theory predicts an increase in the relaxation time by a factor of about 10 . The observed conservation of the surface roughness in the case of PS/PI clearly not explicable by a simple reduction in Laplace pressure. A comparison of the results as a function of molecular weight and interfacial tension showed that enthalpy related effects can remarkably change the relaxation behavior of artificially rough PS surfaces. No such effect was observed, however, by increasing the molecular weight of the polymer and thus the degree of entropic confinement. Therefore, the driving force of the relaxation and enhanced mobility of the near surface region are due to the identical enthalpic origin, interfacial tension.

To conclude, we observed that single asperities of artificially rough polymer surfaces may relax partially at temperatures far below the bulk glass transition temperature. Rate and terminal degree of the relaxation depend strongly on the annealing temperature. A full relaxation is only observable for $T \approx T_{\text {g,bulk }}$ or higher. $A$ set of experiments designed to test whether the observed mobility is of entropic or enthal pic origin indicated that entropy related confinement issues are negligible in comparison to the influence of the enthalpic interfacial interactions. This observation of a structure size dependent mobility due to enthal pic rather than entropic drivers is novel and may provide insight for the theoretical modeling of surface effects on mobility and $T_{g}$ in thin film geometries.

Acknowledgment. T.K. acknowledges the support of the Deutsche Forschungsgemeinschaft. This work was also supported by the U.S. Department of Energy, Office Basic Energy Sciences, under Contract DE-FG0296E R45612 and the National Science Foundation Materials Research Science and E ngineering Center (DMR9809365). 


\section{References and Notes}

(1) Krausch, G. Mater. Sci. Eng. 1995, R14, 1-94.

(2) Budkowski, A. Adv. Polym. Sci. 1999, 148, 1-111.

(3) Binder, K. Acta Polym. 1995, 46, 204-225.

(4) de Gennes, P. G., Sanchez, I. C., Eds.; Butterworth-Heinemann: London, p 55.

(5) Fourche, G. Polym. Eng. Sci. 1995, 35, 957-967.

(6) Klein, J. Science 1990, 250, 640-646.

(7) Klein, J .; Kumacheva, E.; Mahalu, D.; Perahia, D.; Fetters, L. J . Nature 1994, 370, 634-636.

(8) Keddie, J . L.; J ones, R. A. L.; Cory, R. A. Faraday Discuss. 1994, 98, 219-230.

(9) Keddie, J . L.; J ones, R. A. L.; Cory, R. A. Europhys. Lett. 1994, 27, 59.

(10) Forrest, J . A.; Dalnoki-Veress, K.; Stevens, J . R.; Dutcher, J R. Phys. Rev. Lett. 1996, 77, 2002-2005.

(11) Forrest, J . A.; Dalnoki-Veress, K.; Dutcher, J . R. Phys. Rev. E 1997, 56, 5705-5716.

(12) Forrest, J . A.; Mattsson, J . Phys. Rev. E 2000, 61, R53-R56.

(13) DeMaggio, G. B.; Frieze, W. E.; Gidley, D. W.; Zhu, M.; Hristov, H. A.; Yee, A. F. Phys. Rev. Lett. 1997, 78, 15241527.

(14) J ean, Y. C.; Zhang, R.; Cao, H.; Yuan, J .-P.; Huang, C.-M.; Nielsen, B.; Asoka-Kumar, P. Phys. Rev. B 1997, 56, R8459.

(15) Kajiyama, T.; Tanaka, K.; Takahara, A. Macromolecules 1997, 300, 2280-285.

(16) Tanaka, K.; Taura, A.; Ge, S. R.; Takahara, A.; Kajiyama, T. Macromol ecules 1996, 29, 3040-3042.

(17) Tsui, O. K. C.; Wang, X. P.; Ho, J . Y. L.; Ng, T. K.; Xiao, X. Macromol ecules 2000, 33, 4198-4204.
(18) Ge, S.; Pu, Y.; Zhang, W.; Rafailovich, M.; Sokolov, J .; Buenviaje, C.; Buckmaster, R.; Overney, R. M. Phys. Rev. Lett. 2000, 85, 2340-2343.

(19) Liu, Y.; Russell, T. P.: Samant, M. G.; Stühr, J .; Brown, H. R.; Cossy-Favre, A.; Diaz, J . Macromol ecules 1997, 30, 77687771.

(20) J ones, R. A. L. Curr. Opin. Colloid Interface 1999, 4, 153158.

(21) Doruker, P.; Mattice, W. L. Macromolecules 1999, 32, 194198.

(22) de Gennes, P. G. Eur. Phys. J . E 2000, 2, 201-205.

(23) Mayes, A. M. Macromolecules 1994, 27, 3114-3115.

(24) Baschnagel, J .; Binder, K. Macromolecules 1995, 28, 6808.

(25) Müller, M.; Binder, K.; Oed, W. J . Chem. Soc., Faraday Trans. 1995, 91, 2369-2379.

(26) Rouault, Y.; Dünweg, B.; Baschnagel, J .; Binder, K. Polymer 1996, 37, 297-304

(27) Mansfield, K.; Theodorn, D. N. Macromolecules 1991, 24, 6283.

(28) Fox, T.; Flory, P. Polym. Sci. 1954, 14, 315.

(29) Orchard, S. E. Appl. Sci. Res. A 1962, 11, 451-464.

(30) Andrei, D. C.; Hay, J . N.; Keddie, J . L.; Sear, R. P.; Yeates, S. G. J . Phys. D: Appl. Phys. 2000, 33, 1975-1981.

(31) Hamdorf, M.; J ohannsmann, D. J . Chem. Phys. 2000, 112, 4262-4270.

(32) Varnier, F.; Mayani, N.; Rasigni, G. Appl. Opt. 1989, 28, 127. MA0020335 